\title{
In Vitro Antibacterial and Antifungal Potential of Amyrin-Type Triterpenoid Isolated from Datura metel Linnaeus
}

\author{
Sami Bawazeer ${ }^{1}{ }^{1}$ and Abdur Rauf $\mathbb{D D}^{2}$ \\ ${ }^{1}$ Department of Pharmacognosy, Faculty of Pharmacy, Umm Al-Qura University, Makkah, P.O. Box 42, Saudi Arabia \\ ${ }^{2}$ Department of Chemistry, University of Swabi, Swabi, KPK, Pakistan \\ Correspondence should be addressed to Abdur Rauf; mashaljcs@yahoo.com
}

Received 27 May 2021; Accepted 6 September 2021; Published 20 September 2021

Academic Editor: Dorota Formanowicz

Copyright (c) 2021 Sami Bawazeer and Abdur Rauf. This is an open access article distributed under the Creative Commons Attribution License, which permits unrestricted use, distribution, and reproduction in any medium, provided the original work is properly cited.

\begin{abstract}
The aim of the present investigation was to evaluate the effect of the combined crude extract, fractions, and compound 1 isolated from the fruits of Datura metel against selected microbial (bacteria and fungi) strains. Results of antibacterial screening indicated marked susceptibility of the extract and its fractions against tested bacterial strains. Among the extract and various fractions, the chloroform fraction exhibited a significant effect against different bacterial strains including Escherichia coli, Staphylococcus aureus, and Bacillus subtilis with an inhibitory zone ranging from 18 to $24 \mathrm{~mm}$. Similarly, results of antifungal activity revealed that the chloroform fraction displays a promising effect against various fungal strains. The chloroform fraction was subjected to repeated chromatography analysis, which yielded compound 1 (daturaolone). Daturaolone exhibited potent activity against selected bacterial strains including Klebsiella pneumoniae, B. subtilis, S. epidermidis, and S. aureus with an inhibitory zone ranging from 12 to $30 \mathrm{~mm}$. In addition, the extracts and daturaolone exhibited significant sensitivity against T. longifusus, C. albicans, A. flavus, M. canis, F. solani, and C. glabrata. Taken all together, it is concluded that our findings validated the traditional use of $D$. metel to treat various infectious diseases, which is supported by daturaolone.
\end{abstract}

\section{Introduction}

Medicinal plants are the key source of diverse classes of natural products including primary and secondary metabolites, which are produced in plants. Primary metabolites are used by plants for various life functions such as respiration, cell division, growth, reproduction, and photosynthesis among others. On the other hand, secondary metabolites, also known as phytochemicals, natural products, are chemical constitutes which are isolated from plants and utilized for medicinal purposes. In this respect, various plant extracts and phytochemicals are reported for diverse pharmacological potential such as drugs for sexual dysfunction, antioxidants, laxative, antidiabetics, and anticancer agents [1].

Datura metel L belonging to the Solanaceae family is locally known as Dhutura. It is an erect shrub that possesses spreading branches and can reach 1.5 meters in height [2]. D. metel possesses broadly ovate, simple alternative, glabrous, shallowly lobed, and dark green leaves. Its flowers are solitary, large, and trumpet-shaped, have a sweet smell, and have a wide range of color (yellow to dark purple), which appeared in the evening as well as morning. D. metel can be found in tolerate average soil, but it commonly prefers moist soil or alkaline soil. The plant grows in America and is distributed in tropical and subtropical regions, like India and East Asia.

D. metel is used as the traditional Bangladeshi herbal medicine $[3,4]$. In the Chinese folkloric system, D. metel flowers are recognized as baimantuoluo and used in the treatment of skin inflammation and psoriasis. Similarly, $D$. metel seeds are used for the treatment of bronchitis, ulcer, diabetes, and skin rashes. In addition, seeds of $D$. metel are used in the preparation of tea, which is used as sedative, and dried flowers are smoked as cigarettes $[5,6]$. Interestingly, D. metel produces valuable active secondary metabolites including alkaloids, steroids, triterpenoids, flavonoids, saponins, and tannins [7]. The pharmacological applications of $D$. metel are due to the bioactive phytochemicals. In this 
context, the alkaloid scopolamine is an important compound extracted from $D$. metel. Scopolamine has been documented for the treatment of different diseases such as asthma and bronchitis [8]. It is also used to cure fever, insanity, epilepsy, heart disease, diarrhea, and diabetes as well as skin diseases $[8,9]$. It is also an important source of withanolides, which are used in the treatment of hallucinogenic potency as well as pain $[10,11]$. Furthermore, D. metel has been reported to relieve tooth pain [12]. In addition, $D$. metel has several uses in the ayurvedic system; several classes of secondary metabolites from this plant are used to treat skin infection and hair fall [7]. Research findings indicated that various solvent extracts of $D$. metel exhibited documented antibacterial, antifungal, and anti-inflammatory properties $[13,14]$. Similarly, atropine extracted from $D$. metel has been used to dilate the pupil, and it helps in the surgery of eyes [15]. Based on the preceding discussion, this work is aimed at exploring D. metel phytochemically and pharmacologically. In addition, this work deals with the isolation and purification of daturaolone (1), along with its in vitro antibacterial and antifungal activity for the discovery of new antimicrobial drugs.

\section{Material and Methods}

2.1. Collection of the Plant. Fruits of D. metel were collected from the mountain area of Village Razagram Tehsil Khall, District Dir, KPK, Pakistan. Dr. Muhammad Muhammad Ilyas, a botanist at the Department of Botany, University of Swabi, KPK, Pakistan, identified and authenticated the plant. A voucher specimen BOT(UOS-521) was deposited at the herbarium located at the Botany Department, University of Swabi, Pakistan.

2.2. Extraction and Isolation of Daturaolone (1). The collected fruits $(8.00 \mathrm{~kg})$ were washed with water to remove dust particles and then dried in the shade. The dried fruits were ground to fine powder by means of a grinder machine. The powdered material was subjected to cold extraction with commercial grade methanol to afford $349.76 \mathrm{~g}$ crude extract. The crude extract was dried at room temperature and was subjected to successive fractionation with various organic solvent and yielded $52.81 \mathrm{~g}$ from $n$-hexane, $93.61 \mathrm{~g}$ from chloroform, and $45.10 \mathrm{~g}$ from ethyl acetate as per our previous reported method [15]. The chloroform fraction was selected for chromatographic analysis, where the column was eluted with mixtures of $n$-hexane and ethyl acetate $(0: 100)$ with increasing polarity. Two hundred subtractions were collected and compiled based on the TLC profile, which afforded 15 subfractions (SB-1-SB-15). Subfraction SB-7 was subjected to chromatographic analysis to yield white crystals. These crystals were washed with $n$-hexane and regrown in a mixture of $n$-hexane and ethyl acetate, which afforded daturaolone $(2.7 \mathrm{~g})$. The chemical structure of daturaolone (Figure 1) was confirmed with the aid of different spectroscopic techniques and by X-ray crystallography [15].

\subsection{Antimicrobial Screening}

2.3.1. Antibacterial Activity. The crude extract, various solvent extracted fractions, and daturaolone were screened for antibacterial activity according to our recently published protocols $[12,16]$. Bacterial strains used in this investigation were Klebsiella pneumoniae (ATCC 700603), Escherichia coli (ATCC25922), Staphylococcus aureus (ATCC 25923), Bacillus subtilis (ATCC 6633), and Staphylococcus epidermidis (ATCC25925) clinical isolates. These bacterial strains were revived from the PNLR Center, Institute of Chemical Sciences, University of Peshawar, KPK, Pakistan. The selected strains were stored in Mueller Hinton agar in the refrigerator at $4^{\circ} \mathrm{C}$, prior to culture. The screening of extract/fractions and daturaolone was performed using the agar well diffusion methods according to a standard protocol. In this methodology, Mueller Hinton agar (MHA) was used as the medium. All cultures were taken in triplicate, and the incubation was achieved at standard conditions $\left(37^{\circ} \mathrm{C}, 24-72 \mathrm{~h}\right)$. The prepared cultures $(0.6 \mathrm{~mL})$ of the screened organism were placed in a sterile petri dish, and then, $20 \mathrm{~mL}$ of sterile molten Mueller Hinton agar (MHA) was added. Then, a hole $(6 \mathrm{~mm})$ was bored into the medium using a borer. The standard drug (imipenem), extract, fractions, and daturaolone were introduced to the medium in various concentrations. Incubation was performed for $24 \mathrm{~h}$ at $37^{\circ} \mathrm{C}$, and the diameters of the inhibitory zone of bacterial growth were recorded in millimeters.

2.3.2. Antifungal Activity. The crude extract, different extract/fractions, and daturaolone were screened for antifungal activity against the fungal strains Candida albicans, Candida glabrata, Trichophyton longifusus, Aspergillus flavus, Fusarium solani, and Microsporum canis clinical isolates using the tube dilution assay according to a published protocol [17]. According to this assay, a stock solution was prepared by dissolving the extract/fractions and isolated compound (1) in sterile dimethyl sulfoxide (DMSO). A screw-cap tube was taken, and $6 \mathrm{~mL}$ of Sabouraud dextrose was poured in each tube and then put in an autoclave at $20^{\circ} \mathrm{C}$ for 15 minutes; after that, it was cooled to $15^{\circ} \mathrm{C}$. The nonsolidified Sabouraud Dextrose Agar (SDA) was then poisoned with stock solution $(66.8 \mu \mathrm{L})$ which gives the final concentrations of $400 \mu \mathrm{g}$ of the extract per $\mathrm{mL}$ of SDA. Then, tubes were subjected to solidification in the slanted position at low temperature $\left(25^{\circ} \mathrm{C}\right)$. Individually, the tube was then incubated with a piece ( $4 \mathrm{~mm}$ diameter) of inoculums detached from a 7-day old culture of fungi for nonmycelial growth; an agar surface streak was then working. Dimethyl sulfoxide was used as the control while amphotericin and miconazole were used as the reference drug. The inhibition of fungal growth was recorded after 7 days of incubation at $28^{\circ} \mathrm{C}$ and humidity of $40-50 \%$. The test tubes were scrutinized for the visible growth of fungal strain, and then, the percentage inhibitions were determined.

2.4. Statistical Analysis. Assays were conducted in triplicate, and data were subjected to one-way analysis of variance (ANOVA). Results are expressed as the mean \pm standard error of the mean (SEM). Statistical analysis was performed using the GraphPad Prism 7 software (GraphPad Software, 


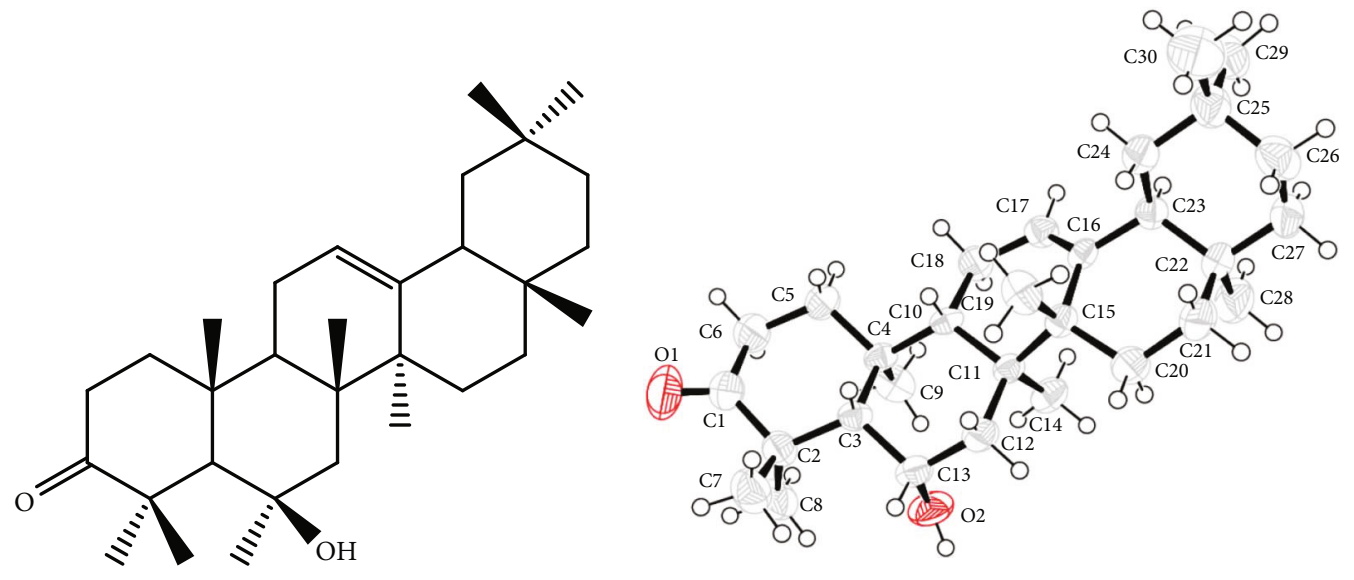

Figure 1: Structure and X-ray crystallographic image of compound 1 isolated from D. metel.

San Diego, California, USA, https://www.graphpad.com); differences were considered significant at $p \leq 0.05$.

\section{Results}

3.1. Effect of the Extract/Fractions against Bacterial Strain. The results of the extract and various isolated fraction of Datura metel Linnaeus fruits against E. coli, K. pneumoniae, S. epidermidis, S. aureus, and B. subtilis are displayed in Tables 1 and 2 . The crude methanolic extract and its subsequent fractions exhibited were found to be sensitive against different Gram-positive and Gram-negative bacterial strains. The chloroform fraction exhibited significant activity against E. coli, S. aureus, and B. subtilis. The methanolic extract showed good activity against E. coli, S. epidermidis, S. aureus, and $B$. subtilis while the ethyl acetate fraction exhibited moderate sensitivity against Escherichia coli, S. epidermidis, S. aureus, and B. subtilis. Among the entire fraction, the hexane fraction did not exhibit impressive activity.

3.2. Effect of Daturaolone against Bacterial Strains. Results of the antibacterial activity of daturaolone isolated from $D$. metel Linnaeus fruits are shown in Tables 1 and 2. Results revealed that daturaolone exhibits potent activity against $B$. subtilis, significant activity against $E$. coli, and good activity against S. epidermidis, S. aureus, and K. pneumoniae.

3.3. Effect of the Extract/Fractions against Fungal Strains. Shown in Table 3 are results of our study of the antifungal activity of the extract and various fractions of D. metel Linnaeus. Results indicated that the chloroform fraction exhibits excellent activity against $A$. flavus, M. canis, and F. solani followed by the methanol extract, which showed good activity against $A$. flavus, $M$. canis, and F. solani. On the other hand, the ethyl acetate fraction was sensitive only against $M$. canis while the $n$-hexane was the least active among all fractions.

3.4. Antibacterial Effect of Daturaolone. Presented in Table 3 are results of our investigation related to the antibacterial effect of daturaolone isolated from $D$. metel. Results reveal that daturaolone exerts significant activity against E. coli, K. pneumoniae, S. epidermis, S. aureus, and B. subtilis.

Our findings indicate that the crude extracts/fraction and isolated daturaolone exhibit a broad-spectrum antimicrobial effect against selected pathogens. It has been widely perceived that irrational use of antibiotics could lead to the production of pathogenic bacteria with increasing resistance to different classes of antibiotics. Unfortunately, this has led to progressive loss in the therapeutic efficiency of antibiotics at a rate that varies with complexity of required mutation, the ease of clonal spread, or the rate of horizontal gene transfer [18]. In this context, prevalence of infection due to a multidrug resistance pathogen has shown a dramatic increase around the globe [19]. Several novel antibiotics have been discovered, which are used to combat various types of bacterial infections caused by numerous bacteria, which developed resistance to those antibiotics. In this regard, medicinal plants have been considered a rich source of therapeutic agents for thousands of years [20]. A remarkable number of modern medicine having antimicrobial potency has been prepared from various plants. It has been assumed that plant-based antimicrobial agents may have several possible novel mechanisms of action, which should be important in the prevention/reduction on antimicrobial resistance [21].

In addition, our results demonstrated that the methanol extract and its fractions exhibit promising activity against selected bacteria including S. epidermidis, E.coli, S. aureus, and $B$. subtilis, while the ethyl acetate fraction exhibited moderate sensitivity against $S$. epidermidis, E. coli, S. aureus, and B. subtilis. Similarly, daturaolone exerted potency against tested bacterial strains. The chloroform fraction showed excellent effect against E. coli, S. aureus, and B. subtilis with zones of inhibition ranging from 18 to $24 \mathrm{~mm}$. On the other hand, the $n$-hexane fraction did not exhibit considerable activity. Intestinally, daturaolone exhibited potent activity against $B$. subtilis, significant activity against E. coli, and good activity against $S$. epidermidis, $S$. aureus, and $K$. pneumoniae with zone inhibition ranging from 12 to $30 \mathrm{~mm}$.

Similarly, there is a considerable growth in the number of fungus-infected patients throughout the globe; there have 
Table 1: Antibacterial effect (zone of inhibition in $\mathrm{mm}$ ) of the crude extract/fractions and daturaolone isolated from $D$. metel fruits.

\begin{tabular}{lccccccc}
\hline \multirow{2}{*}{ Bacteria species } & & \multicolumn{3}{c}{ Zone of inhibition in mm } \\
& Control & Hexane & Chloroform & EtOAC & Methanol & Daturaolone & Imipenem \\
\hline E. coli & $0 \pm 0.00$ & $0 \pm 0.00$ & $18 \pm 1.44$ & $12 \pm 1.46$ & $16.2 \pm 1.23$ & $25.05 \pm 0.18$ & $28.24 \pm 0.08$ \\
K. pneumoniae & $0 \pm 0.00$ & $0 \pm 0.00$ & $0 \pm 0.00$ & $0 \pm 0.00$ & $0 \pm 0.00$ & $12.45 \pm 00$ & $32.66 \pm 0.09$ \\
S. epidermis & $0 \pm 0.00$ & $0 \pm 0.00$ & $0 \pm 0.00$ & $12.98 \pm 1.08$ & $18.21 \pm 1.90$ & $18.98 \pm 0.40$ & $30.76 \pm 0.12$ \\
S. aureus & $0 \pm 0.00$ & $8.01 \pm 1.44$ & $18.65 \pm 1.40$ & $10.34 \pm 1.44$ & $13.80 \pm 1.34$ & $20.32 \pm 0.38$ & $32.87 \pm 0.18$ \\
B. subtilis & $0 \pm 0.00$ & $6.08 \pm 1.20$ & $24.29 \pm 1.48$ & $8.65 \pm 1.49$ & $10.21 \pm 1.98$ & $30.08 \pm 0.39$ & $34.87 \pm 0.30$ \\
\hline
\end{tabular}

Data are presented as the mean \pm SEM of three sets of individual assays in every column. Standard (imipenem $1 \mathrm{mg}$ ), well size $6 \mathrm{~mm}$, and tested sample $(2 \mathrm{mg} / \mathrm{mL})$.

TABLE 2: The MIC of the crude extract, various fractions, and daturaolone isolated from $D$. metel fruits.

\begin{tabular}{|c|c|c|c|c|c|c|c|}
\hline \multirow{2}{*}{ Bacteria species } & \multicolumn{7}{|c|}{$\mathrm{MIC}(\mu \mathrm{g} / \mathrm{mL})$} \\
\hline & Control & $n$-Hexane & Chloroform & EtOAC & Methanol & Daturaolone & Imipenem \\
\hline E. coli & - & - & $143.21 \pm 2.00$ & - & $160.23 \pm 2.65$ & $2.39 \pm 1.00$ & $0.18 \pm 0.00$ \\
\hline K. pneumoniae & - & - & - & - & - & - & $0.19 \pm 0.04$ \\
\hline S. epidermis & - & - & - & - & $153.21 \pm 2.54$ & $28.22 \pm 1.88$ & $0.20 \pm 0.06$ \\
\hline S. aureus & - & - & $144.32 \pm 2.01$ & - & - & $20.11 \pm 1.23$ & $0.19 \pm 0.02$ \\
\hline B. subtilis & - & - & $12.98 \pm 1.64$ & - & - & $0.43 \pm 0.34$ & $0.21 \pm 0.04$ \\
\hline
\end{tabular}

Results are expressed as the mean \pm SEM of three sets of individual assays in every column.

TABLE 3: Antifungal effect (zone of inhibition in $\mathrm{mm}$ ) of the crude extract, different fractions, and daturaolone isolated from $D$. $m e t e l$ fruits.

\begin{tabular}{|c|c|c|c|c|c|c|c|}
\hline \multirow{2}{*}{ Fungal species } & \multicolumn{7}{|c|}{$\%$ zone of inhibition } \\
\hline & $n$-Hexane & Chloroform & EtOAC & Methanol & Daturaolone & Standard & MIC $(\mu \mathrm{g} / \mathrm{mL})$ \\
\hline T. longifusus & - & - & - & - & - & Miconazole & $108.23 \pm 1.21$ \\
\hline C. albicans & - & - & - & - & - & Miconazole & $21.56 \pm 1.15$ \\
\hline A. flavus & - & $24.23 \pm 2.88$ & - & $20.20 \pm 2.96$ & $36.77 \pm 1.10$ & Amphotericin B & $98.98 \pm 0.80$ \\
\hline M. canis & $12.87 \pm 2.87$ & $28.98 \pm 2.00$ & $16.23 \pm 1.87$ & $25.32 \pm 1.45$ & $38.98 \pm 1.00$ & Miconazole & $73.34 \pm 0.64$ \\
\hline F. solani & - & $22.98 \pm 1.98$ & - & $16.11 \pm 2.88$ & $32.98 \pm 1.60$ & Miconazole & $110.12 \pm 0.23$ \\
\hline C. glabrata & - & - & - & - & - & Miconazole & $108.23 \pm 0.06$ \\
\hline
\end{tabular}

Results are expressed as the mean \pm SEM of three sets of individual assays in every column.

been prominent deviations in fungal pathogens. The greatest potential description of these adjustments is the appearance of new fugal pathogens and the growing range with more infections due to fungal pathogens. Possibly, these properties are associated with the increase in the number of patients who are extremely vulnerable to fungal infection. These infections regularly occur in patients with surgery, bone narrow transplant, cancer, and other immunosuppressed patients maximum especially those infected with human immunodeficiency virus (HIV) [21]. In this study, we have screened the extract/fractions and daturaolone for activity against selected fungal strains. Our findings showed that the chloroform fractions exhibited excellent activity against A. flavus, M. canis, and F. solani followed by the methanol extract, which showed good activity against $A$. flavus, $M$. canis, and F. solani. On the other hand, the ethyl acetate fraction was sensitive only against $M$. canis while the $n$-hexane was the least active among all fractions. Daturao- lone exhibited significant activity against T. longifusus, $C$. albicans, A. flavus, M. canis, F. solani, and C. glabrata as compared to the standard drug (Table 3 ). Based on these results, this plant is recommended for further studies that could include isolation of novel natural products as well as derivatization and biotransformation of daturaolone in order to discover novel compounds of clinical applications.

\section{Conclusions}

In summary, findings from this investigation suggest that the fruit crude extracts from $D$. metel and its various fractions exhibit a remarkable and promising antibacterial effect against selected bacterial strains and thus justify the traditional use of the plant for the treatment of numerous diseases. Additionally, the plant extracts exhibited excellent antifungal activity. Furthermore, our findings showed that daturaolone isolated from this medicinal plant exhibited 
promising antimicrobial activity and could be a lead for the discovery of new antibacterial and antifungal agents. However, more detailed studies are required to establish the safety and efficacy of this plant and to isolate more bioactive compounds.

\section{Data Availability}

The data associated with this paper are given in the main text of this paper. The spectroscopic data of compounds is available from corresponding authors upon request.

\section{Conflicts of Interest}

The authors declare no conflict of interest.

\section{Authors' Contributions}

Sami Bawazeer and Abdur Rauf designed this study. Abdur Rauf did the work pertaining to plant collection, extraction, isolation, and biological screening. The three authors jointly wrote and edited this manuscript and approved submission.

\section{Acknowledgments}

This research project was funded by grant number 14-MED333-10 from National Science, Technology and Innovation Plan (MAARIFAH), King Abdulaziz City for Science and Technology (KACST), Saudi Arabia. We are also grateful to Umm Al-Qura University for their logistic support.

\section{References}

[1] S. Bawazeer and A. Rauf, "In vivo anti-inflammatory, analgesic, and sedative studies of the extract and naphthoquinone isolated fromDiospyros kaki(Persimmon)," ACS Omega, vol. 6, no. 14, pp. 9852-9856, 2021.

[2] M. M. Khaton and M. M. Shaik, "Review on Datura metel: a potential medicinal plant," Global Journal of Research on Medicinal Plants and Indigenous Medicine, vol. 1, no. 4, pp. 123-132, 2012.

[3] R. Dabur, M. Ali, H. Singh, J. Gupta, and G. L. Sharma, "A novel antifungal pyrrole derivative from Datura metel leaves," Die Pharmazie-An International Journal of Pharmaceutical Sciences, vol. 59, pp. 568-570, 2004.

[4] R. S. Sangwan and H. Camefort, "The tonoplast, a specific marker of embryogenic microspores of Datura cultured in vitro," Histochemistry, vol. 78, no. 4, pp. 473-480, 1983.

[5] D. E. Okwu and E. C. Igara, "Isolation, characterization and antibacterial activity of alkaloid from Datura metel Linn leaves," African Journal of Pharmacology, vol. 3, pp. 277-281, 2009.

[6] R. N. Chopra, S. N. Nayar, and I. C. Chopra, Glossary of Indian Medicinal Plants, Council of Scientific and Industrial Research, New Delhi, India, 1956.

[7] P. Soni, A. A. Siddiqui, J. Dwivedi, and V. Soni, "Pharmacological properties of Datura stramonium L. as a potential medicinal tree: An overview," Asian Pacific Journal of Tropical Biomedicine, vol. 2, no. 12, pp. 1002-1008, 2012.
[8] B. Y. Yang, R. Guo, T. Li et al., "New anti-inflammatory withanolides from the leaves of Datura metel L.," Steroids, vol. 87, pp. 26-34, 2014.

[9] R. N. Chopra, S. L. Nayar, and L. C. Chopra, Glossary of Indian Medicinal Plants, Council of Scientific and Industrial Research, New Delhi, India, 1986.

[10] M. Abubakar, U. Suleiman, A. Frank, and A. Ukwuani, "Hallucinogenic effects of aqueous seeds extract of Datura metel in rats," Internet Journal of Pharmacology, vol. 9, no. 1, p. 1, 2010.

[11] T. N. Arjun, H. Sudhir, E. Saxena, A. Dayma, R. S. Raghuvanshi, and R. Shah, "Role of Siddha system of medicine in the management of oro-facial diseases," World Journal of Pharmaceutical Sciences, vol. 4, no. 8, pp. 1661-1671, 2015.

[12] A. Rauf, G. Uddin, H. Khan, M. Arfan, and B. S. Siddiqui, "Bioassay-guided isolation of antibacterial constituents fromDiospyros lotusroots," Natural Product Research, vol. 30, no. 4, pp. 426-428, 2016.

[13] T. H. A. Alabri, A. H. S. Al Musalami, M. A. Hossain, A. M. Weli, and Q. Al-Riyami, "Comparative study of phytochemical screening, antioxidant and antimicrobial capacities of fresh and dry leaves crude plant extracts of Datura metel L," Journal of King Saud University, vol. 26, no. 3, pp. 237-243, 2014.

[14] F. C. Akharaiyi, "Antibacterial, phytochemical and antioxidant activities of Datura metel," International Journal of PharmTech Research, vol. 3, no. 1, pp. 478-483, 2011.

[15] K. Wang, H. Sun, B. Wu, and Y. Pan, "Two novel Olean triterpenoids fromCelastrus hypoleucus," Helvetica Chimica Acta, vol. 88, no. 5, pp. 990-995, 2005.

[16] A. Rauf, S. Uysal, T. Ben Hadda et al., "Pakistan'dan toplanan üç tıbbi bitkinin antibakteriyel etkileri, sitotoksisite ve fitotoksisite profilleri," Marmara Pharmaceutical Journal, vol. 21, no. 2, pp. 261-268, 2017.

[17] G. Uddin, A. Rauf, B. S. Siddiqui, and H. Khan, “The biological screening of extracts/fractions of various parts of Pistacia integerrima Stewart against pathogenic fungi," Translational Medicine, vol. 4, pp. 120-125, 2013.

[18] D. King, L. W. Cheever, A. Hood, T. D. Horn, M. G. Rinaldi, and W. G. Merz, "Primary invasive cutaneous Microsporum canis infections in immunocompromised patients," Journal of Clinical Microbiology, vol. 34, no. 2, pp. 460-462, 1996.

[19] S. H. Eom, D. H. Kim, S. H. Lee et al., "In vitro antibacterial activity and synergistic antibiotic effects of phlorotannins isolated from Eisenia bicyclis against methicillin-resistant Staphylococcus aureus," Phytotherapy Research, vol. 27, no. 8, pp. 1260-1264, 2013.

[20] F. Israr, F. Hassan, B. Naqvi, I. Azhar, S. Jabeen, and S. Hasan, "Report: studies on antibacterial activity of some traditional medicinal plants used in folk medicine," Pakistan Journal of Pharmaceutical Sciences, vol. 25, no. 3, pp. 669-674, 2012.

[21] C. Walter, Z. K. Shinwari, I. Afzal, and R. N. Malik, "Antibacterial activity in herbal products used in Pakistan," Pakistan Journal of Botany, vol. 43, pp. 155-162, 2011. 